\title{
BIOMECHANICAL EVALUATION OF DOUBLE Y-SHAPED VERSUS CONVENTIONAL STRAIGHT TITANIUM MINIPLATES FOR THE TREATMENT OF MANDIBULAR ANGLE FRACTURES
}

\author{
Atef M Hasanein* and Abdelmageed Alfakhrany**
}

\begin{abstract}
Aim of this experimental study: Was to test the resistance to mechanical force of a double Y- shaped titanium miniplate versus conventional straight titanium miniplate for the treatment of mandibular angle fracture.

Materials and methods: Ten fresh sheep hemi mandibles, sectioned at the angle region, were used to evaluate two plating techniques. One group (Group A) received fixation via a double Yshaped titanium miniplate and the second group (group B) via a six-hole conventional straight non-compression titanium miniplate .Each hemi mandible in group A and group B was subjected to tensile forces using universal testing machine.
\end{abstract}

Results: During the test, the group (A) fixed by double Y-shaped miniplate, the plates were fractured in all group samples at mean of the Yield load $(745 \mathrm{~N})$. The mean degree of displacement of this group was $7.8 \mathrm{~mm}$ ). While in group (B), the conventional straight titanium mini plates were fractured at force (the mean; $665.6 \mathrm{~N}$ ). The mean of degree of displacement of this group was $7.8 \mathrm{~mm})$

Conclusion; Double Y-shaped miniplate with 6 holes and $9.0 \mathrm{~mm}$ spacing had greater resistance to displacement and provided more favorable biomechanical behavior than the conventional straight miniplates in the repair of mandibular angle fractures.

KEYWORDS: double Y shaped plate; angular fractures; conventional straight mini plate.

\section{INTRODUCTION}

Mandibular fractures are one of the most common facial skeletal injuries. They can be caused by road traffic accidents, assaults, industrial injuries, falls or sports injuries, but the relative number of each varies considerably between countries and areas. Despite the advances and studies on the type of internal fixation used for treatment of fractures of the mandibular angle, these fractures still present unpredictable results and difficulties in treatment in relation to other mandibular fractures, leading to discussions on the ideal treatment. ${ }^{1-3}$

\footnotetext{
* Lecturer, Oral and Maxillofacial Surgery, Faculty of Dental Medicine, Al-Azhar University Cairo, (Boys) Egypt
} 
Various techniques of internal fixation have been studied with great variation in rates of complications. Lag screws reconstruction plates, dynamic compression plates, miniplates with monocortical screws used according to the Champy philosophy or AO/ASIF philosophy, and locking plates, can be used for this type of fracture fixation..$^{4-7}$

Miniplates have been used to facilitate stability between bony fragments in the maxillofacial region and are currently the preferred surgical method for the fixation of fractures and osteotomies. The advantages of using miniplates include easy handling and easy plate contouring and adaptation to the bone). ${ }^{5}$ Miniplate osteosynthesis ensures adequate fracture stability, improves bone healing, and allows early functional mobilization). ${ }^{6}$ During fracture treatment planning, important decisions that must be made include determining the best position, orientation, and plate type and material. The first and foremost consideration is the rigidity of the repaired fracture section, and the second pertains to the level of stress generated in the miniplates under bite forces) ${ }^{7,8}$.

A variety of miniplates have been used to repair mandibular angle fractures. In the original Champy version, a 4-hole miniplate without center space was used). ${ }^{9}$ Today, although this type of plate is still applicable, alternatives that provide similar or incrementally higher stability have emerged.

Therefore, the aim of this experimental biomechanical study was to test the resistance to mechanical force of a double Y- shaped titanium miniplate versus conventional straight titanium miniplate for the treatment of mandibular angle fracture in healthy sheep.

\section{MATERIALS AND METHODS}

Ten fresh sheep hemi mandibles of healthy sheep of a similar age group (8-10 months of age) and fed on the same diet were the model samples of this experimental study. All mandibles were stripped of their soft tissues and sectioned at the midline by using a diamond disk. The osteotomy line has been extended from concavity connecting ramus and body superiorly to the convexity inferiorly to create a classic angular fracture. All prepared model specimens were equally and randomly divided into two groups according to the method of fixation used.

One group (Group A) received fixation via a double Y- shaped titanium miniplate (StrykerLeibenger, Germany) in fig 1 and the second group (group B) via a six-hole conventional straight noncompression titanium miniplate (Stryker-Leibenger, Germany) fig 2. .Each hemi mandible in group A and group B was subjected to tensile forces using universal testing machine. All used fixation screws were fixed bicortically in both groups.

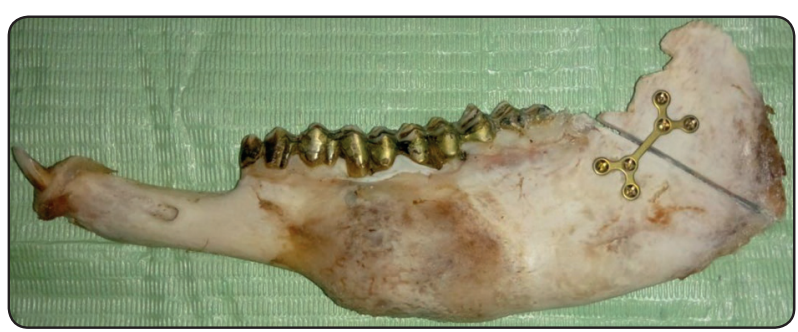

Fig. (1) Double Y- shaped titanium miniplate fixation in prepared model specimen in group A



Fig. (2) Six-hole conventional straight non-compression titanium miniplate fixation in prepared model specimen in group B

All the groups' hemi mandibles were submitted to tensile loading tests using the universal testing machine. The forces applied by the masticatory muscles of human were simulated in vitro by this machine. The fixed hemi mandibles were positioned in such a way as to permit an examination of the 
maximal resistance by the fixation methods during simulated forces of mastication.

The LLOYD LR- $5 \mathrm{kN}$ model of the Lloyd Universal Mechanical Testing Machine (Lloyd Instruments LTD, Segensworth, Fareham, England), is the equipment used for load testing and it was connected to a computer with a specialized software.

The testing loads were progressively applied at a constant head speed of $10 \mathrm{~mm} / \mathrm{min}$ and with forces measured in (Newton) and all the obtained data were transferred and recorded with the attached computer.



Fig. (3) Prepared model specimen from group A fixed the testing machine

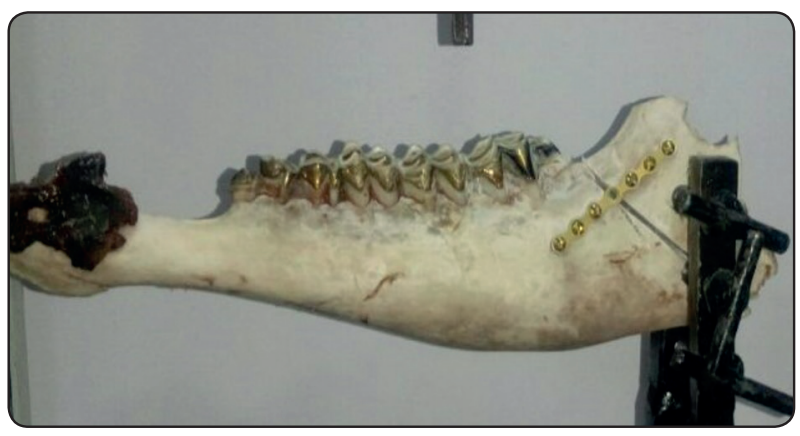

Fig. (4) Prepared model specimen from group B fixed the testing machine

\section{RESULTS}

The aim of the current study was used to evaluate the biomechanics of the double Y-shaped versus conventional straight titanium mini plates in treatment of angular fracture of the mandible. During the test, the group (A) fixed by double Y-shaped miniplate, the plates were fractured in all group samples at mean of the Yield load $(745 \mathrm{~N})$.
The mean degree of displacement of this group was $7.8 \mathrm{~mm}$ ). While in group (B), the conventional straight titanium mini plates were fractured at force (the mean; 665.6N). The mean of degree of displacement of this group was $7.8 \mathrm{~mm}$ ) as seen in fig 5 .

In comparison between the groups according to yield load values as shown by the print out chart, groups showed statistically significant difference in mean yield load as shown in table 1 .

TABLE (1): The means, standard deviation (SD) values and results of Student's t-test for the comparison between mean yield load of the 2 groups.

\begin{tabular}{|c|c|c|c|c|}
\hline \multicolumn{2}{|c|}{ Group A } & \multicolumn{2}{c|}{ Group B } & \multirow{2}{*}{$P$-value } \\
\hline Mean & SD & Mean & SD & \multirow{2}{*}{$<0.007 *$} \\
\cline { 1 - 4 } 745 & 19.29 & 665.6 & 19.8 & \\
\hline
\end{tabular}

*Significant at $P \leq 0.05$

The yield displacement values as shown by the print out chart, groups showed no statistically significant difference in mean yield displacement as shown in table 2 .

TABLE (2): The means, standard deviation (SD) values and results of Student's t-test for the comparison between mean yield displacement of the 2 groups.

\begin{tabular}{|c|c|c|c|c|}
\hline \multicolumn{2}{|c|}{ Group A } & \multicolumn{2}{c|}{ Group B } & \multirow{2}{*}{ P-value } \\
\hline Mean & SD & Mean & SD & \multirow{2}{*}{$0.409 *$} \\
\hline 7.8 & 0.27 & 7.8 & 0.44 & \\
\hline
\end{tabular}

*Significant at $P \leq 0.05$

The stiffness values as shown by the print out chart, groups showed statistically significant difference in mean yield stiffness as shown in table 3 
TABLE (3): The means, standard deviation (SD) values and results of Student's t-test for the comparison between mean yield stiffness of the 2 groups.

\begin{tabular}{|c|c|c|c|c|}
\hline \multicolumn{2}{|c|}{ Group A } & \multicolumn{2}{c|}{ Group B } & \multirow{2}{*}{ P-value } \\
\cline { 1 - 4 } Mean & SD & Mean & SD & \multirow{2}{*}{$<0.007^{*}$} \\
\cline { 1 - 3 } 95.35 & 2.42 & 86 & 3.25 & \\
\hline
\end{tabular}

*Significant at $P \leq 0.05$



Fig 5: Samples from group A and B showed plating system fractured after loading

\section{DISCUSSION}

Fracture of the mandible at the angle is common and is difficult to treat, as there is no universal standard protocol to treat angle fractures. Various types of plates have been designed, claiming to be superior to other types in terms of stability and complications. ${ }^{10}$

Different shapes of titanium mini plates have been used widely by surgeons for fixation of the mandibular angular region. ${ }^{9}$

Many of the researches about using semi rigid fixation by using different shaped mini-plate and micro-plate systems in mandibular fracture indicated the plates should be strong yet malleable facilitating stabilization both at superior and inferior borders. So the conclusion, the single mini titanium plates can be used as an alternative to conventional mini plates but contraindicated its use in fractures with less inter fragmentary bone contact at the fracture site. ${ }^{11}$

Although the progress in internal fixation for mandibular fracture during previous decades, it is still needed to understand the behavior of fixation devices in mandibular tension zones as the angle region. ${ }^{12-14}$

Therefore, the current study stressed on evaluating the biomechanical stability characteristics of titanium miniplates of varied shapes. The two types of miniplate shapes were selected for this study because of the frequency of their use. To ensure that only the biomechanical properties of the specific miniplate were tested in this study more accurately, only one miniplate fixation technique was performed.

In the classic Champy technique, the use of a single titanium miniplate with one mm thickness for the fixation of mandibular fractures was reportedly sufficient and accepted as rigid enough. ${ }^{15}$

The goal of this study was to biomechanically evaluate the behavior of the double Y-shaped versus conventional straight titanium mini plates used for fixation of linear angular mandibular fractures under tension loading conditions that mimic the usual physiologically occlusal and muscular stresses.

The estimated maximal bite forces of adult human man could be reached between 300 and 400 Newtons. ${ }^{11,14}$ When a fracture has occurred, force will be reduced in the masticatory system. So, when attempting to test the biomechanics of various fixation techniques, it is important to consider clinically relevant parameters to provide meaningful information to the clinician. ${ }^{12-15}$

In this study, resistance force was recorded and applied at a point $1 \mathrm{~cm}$ distally to the created fracture line of the mandible. This point of force application was selected because it was far enough from the dentate region of the mandible and that loading should not be located within the fracture zone. 
The results of this study showed that in comparison between the two groups according to yield load values, groups showed statistically significant difference in the mean yield load. Group A specimens showed statistically significantly higher mean yield load value than Group B. The reason may be stand behind this result, the distribution of fixed screws in Y shaped fashion may have the more resistance to the applied force.

In comparison between two groups according to the yield displacement values as shown by the print out chart, groups showed no statistically significant difference in mean yield displacement. This may be due to the buttressing effect of the fractured segments which resist the displacement against the applied forces.

According to the stiffness values as shown by the print out chart, groups showed statistically significant difference in mean yield stiffness. In which group A specimens showed statistically significantly higher mean yield stiffness value than group B. double Y shaped plate doesn't have a hole in the connecting bar between the two V shaped parts, this may the reason of its stiffness the conventional plate which has a hole in connecting bar.

\section{CONCLUSION}

Double Y-shaped miniplate with 6 holes and 9.0 $\mathrm{mm}$ spacing had greater resistance to displacement and provided more favorable biomechanical behavior than the conventional straight miniplates in the repair of mandibular angle fractures.

\section{REFERENCES}

1- Erol B, Tanrıkulu R, Go“rgu“n B. Maxillofacial fractures. Analysis of demographic distribution and treatment in 2901 patients (25-year experience). J Craniomaxillofac Surg 2004;32:308-13.

2- Barry CP, Kearns GJ: Superior border plating technique in the management of isolated mandibular angle fractures: A retrospective study of 50 consecutive patients. J Oral Maxillofac Surg 65:1544, 2007
3- Hochuli E, Ha T, Pereira V, Landes C. Use of rectangular grid miniplates for fracture fixation at the mandibular angle. J Oral Maxillofac Surg 2011;69:1436-41.

4- Fox A, Kellman R. Mandibular angle fractures: twominiplate fixation and complications. Arch Facial Plast Surg 2003;5:464-9.

5- De Melo W, Antunes A, Sonoda C, et al. Mandibular angle fracture treated with new three-dimensional grid miniplate. J Craniofac Surg 2012;23:416-7.

6- DandaA.Comparison of a single noncompression miniplate versus 2 noncompression miniplates in the treatment of mandibular angle fractures: a prospective, randomized clinical trial. J Oral Maxillofac Surg 2010;68:1565-7.

7- Barry C, Kearns G. Superior border plating technique in the management of isolated mandibular angle fractures: a retrospective study of 50 consecutive patients. J Oral Maxillofac Surg 2007;65:1544e9.

8- Kalfarentzos E, Deligianni D, Mitros G, Tyllianakis M. Biomechanical evaluation of plating techniques for fixing mandibular angle fractures: the introduction of a new 3D plate approach. Oral Maxillofac Surg 2009;13:139-44.

9- Feledy J, Caterson E, Steger S, Stal S, Hollier L. Treatment of mandibular angle fractures with a matrix miniplate: a preliminary report. Plast Reconstr Surg 2004;114:1711-6.

10- Alkan A, Celebi N, Ozden B, Bas B, Inal S. Biomechanical comparison of different plating techniques in repair of mandibular angle fractures. Oral Surg Oral Med Oral Pathol Oral Radiol Endod 2007;104:752-6.

11. Lakshmi G.,VivekanandS.Three dimensional bone plating system in the management of mandibular fracture. Ann. Essen.Dent.2012; 4:104-10.

12. Eduardo H, Thi K,Valfrido A, Constantin A. Use Of Rectangular Grid Miniplates for Fracture Fixation at the Mandibular Angle. J Oral MaxillofacSurg 2011; 69:143641.

13. Ji B, Wang C, Song F, et al. A new biomechanical model for evaluation of fixation systems of maxillofacial fractures. $\mathrm{J}$ Craniomaxillofac Surg 2012;40:405-8.

14. Al-Moraissi A, El-Sharkawy T, El-Ghareeb T. Three dimensional versus standard miniplate fixation in the management of mandibular angle fractures: a systematic review and meta-analysis. Int $\mathrm{J}$ Oral Maxillofac Surg.2014;43: 708-16.

15- Haug R, Peterson G,Goltz M. A biomechanical evaluation of mandibular angle fracture plating techniques. J Oral MaxillofacSurg 2002;60:73-80. 